\title{
IMPLICACIONES NEUROPSICOLÓGICAS, NEUROLÓGICAS Y PSIQUIÁTRICAS EN UN CASO DE INTOXICACIÓN POR ESCOPOLAMINA
}

\author{
NEUROPSYCHOLOGICAL, NEUROLOGICAL AND PSYCHIATRIC IMPLICATIONS IN A \\ CASE OF POISONING SCOPOLAMINE
}

ANA BERNAL ${ }^{*}$, DAISY GÓMEZ ${ }^{* *}$, SANDRA LÓPEZ ${ }^{* * *}$, MARÍA ROCÍO ACOSTA $^{* * * *}$

UNIVERSIDAD DE SAN BUENAVENTURA, BOGOTÁ - COLOMBIA

FECHA RECEPCIÓN: 20/1/2013・ FECHA ACEPTACIÓN: 1/3/2013

\begin{abstract}
Resumen
En los últimos años se han incrementado los casos de intoxicación por escopolamina con fines delictivos, con importantes repercusiones médicas, cognitivas y sociales, que requieren ser estudiadas, por ello, y ante la escasa investigación realizada en este tema, el objetivo del presente estudio es registrar las implicaciones neurológicas, neuropsicológicas y psiquiátricas en un caso de intoxicación por escopolamina mediante un diseño de caso único basado en el reporte de un hombre de 43 años de edad quien fue expuesto a una intoxicación por escopolamina cuatro meses previos a la valoración. Se realizó la valoración de la ejecución cognitiva del sujeto frente a su coeficiente intelectual y áreas relacionadas con su atención, memoria de trabajo, praxias, lenguaje, memoria, regulación emocional y funciones ejecutivas. También se aplicó la prueba de personalidad MMPI con el fin de ampliar información sobre aspectos de la personalidad del sujeto o alteraciones psiquiátricas derivadas de la intoxicación. Entre los resultados se hallaron dificultades en atención, memoria, control emocional y funciones ejecutivas; además se encontraron consecuencias psiquiátricas y neurológicas, evidenciando que la exposición a un agente tóxico que bloquee el sistema colinérgico, en especial los canales muscarinicos, puede causar la exacerbación de una enfermedad psiquiátrica premórbida.

Palabras clave: derivados de escopolamina, neuropsicología, receptores muscarínicos y trastornos mentales.
\end{abstract}

Abstract
In recent years scopolamine intoxication cases for criminal purposes has increased, with important medical, cognitive and social sequels, that need to be studied, therefore, and given the limited research on this topic, the aim of this study was to record together the implications neurological, neuropsychological and psychiatric case of poisoning scopolamine using a single case design based on the report of scopolamine intoxication about five months of a 43 year old. For this assessment was made of the subject's cognitive performance in front of their IQ and related areas with attention, working memory, praxis, language, memory, emotion regulation and executive functions. Also applied MMPI personality test to expand the information about the subject's personality or psychiatric disorders resulting from intoxication. Among

* Universidad de San Buenaventura, Bogotá, Facultad de Psicología. Especialización en Evaluación y Diagnóstico Neuropsicológico. anam.bernal@gmail.com

** Universidad de San Buenaventura, Bogotá, Facultad de Psicología. Especialización en Evaluación y Diagnóstico Neuropsicológico. daisymarili@hotmail.com *** Universidad de San Buenaventura, Bogotá, Facultad de Psicología. Especialización en Evaluación y Diagnóstico Neuropsicológico. slilianlm16@gmail.com

**** Universidad de San Buenaventura, Bogotá, Facultad de Psicología. Coordinadora Línea de Investigación en Daño Cerebral. Programas de Especialización y Maestría en Neuropsicología. macosta@usbbog.edu.co 
the results there were found difficulties in attention, memory, emotional control and executive functions, and is a high relationship between psychiatric and neurological area, which explains that exposure to a toxic agent that blocks the cholinergic system, especially the channels muscarinic, can cause exacerbation of premorbid psychiatric illness.

Keywords: scopolamine derivatives, neuropsychology, receptor muscarinics and mental disorders.

\section{Introducción}

En Colombia es utilizada de manera habitual la escopolamina con fines delictivos, debido a los efectos inmediatos de esta droga; en donde la víctima entra en estado de pasividad y actitud complaciente, la cual es aprovechada por el delincuente para robarle e inducirle a realizar acciones que pueden lesionarle física, moral o económicamente. Actualmente se utiliza la mezcla de escopolamina con otras sustancias, lo cual hace que se desencadene una sintomatología de mayor gravedad y sus efectos puedan ser permanentes.

Un estudio realizado por Uribe, Moreno, Zamora y Acosta (2005) muestra que a pesar de que en Colombia no existe un consolidado nacional sobre los índices de intoxicación por escopolamina, se han descrito cifras elevadas en los hospitales nacionales; por ejemplo, en Bucaramanga, se reportó que $80 \%$ de las intoxicaciones atendidas en los servicios de urgencias corresponden a escopolamina siendo más frecuente en hombres en edades entre los 20 y 50 años de edad. En el 65\% de los casos se evidenció la aplicación de un solo tóxico, y en el 14.42\% de dos tóxicos, y en el 94\% de los casos fue utilizado con fines de robo. Esto contrasta con las cifras presentadas en investigaciones realizadas en Estados Unidos, en donde en el 79\% de los casos reportados fueron dados secundarios a causas accidentales (Gupta, Peshin, Srivastava \& Kaleekal, 2003).

Habitualmente, los efectos de la intoxicación desaparecen en 48 horas, si el diagnóstico es precoz y no existe retraso en el tratamiento y son raros los casos con secuelas a largo plazo; sin embargo, estas secuelas pueden llegar a ser permanentes dependiendo de la dosis, si la sustancia se administra sola o combinada y de los antecedentes médicos y psicológicos previos de la víctima (Córdoba, 2006; Vanderhoff \& Mosser, 1992). Por lo tanto el interés de la presente investigación es ahondar sobre los efectos neurológicos, neuropsicológicos y psiquiátricos que pueden aparecer posteriormente a la aplicación de una sobredosis.
La escopolamina, según Ardila, Moreno y Gómez (2006) es un alcaloide que se obtiene del procesamiento químico de las semillas de las plantas del genero brugmansia, familia de las solanáceas. Popularmente la planta se conoce como «borrachero» y su fruto como «cacao sabanero» y está compuesta por unas 30 semillas y cada una de estas contiene suficiente escopolamina para intoxicar a un adulto. Después de su procesamiento se convierte en un polvo fino, cristalino, de color blanco, inodoro y de sabor amargo (Álvarez, 2008).

Por lo general, los alcaloides se absorben rápidamente en el tracto gastrointestinal y la vía de administración más frecuente es la oral, aunque también es factible el ingreso de esta sustancia al cuerpo por medio de la vía inhalatoria, táctil o intravenosa, por lo que es fácilmente administrable en bebidas, alimentos, cigarrillos, alimentos, aerosoles e inyecciones.

\section{Implicaciones neurológicas}

Los síntomas presentados en el sujeto posterior a la intoxicación por escopolamina suelen dividirse en periféricos y centrales. Entre los periféricos están la disminución de la secreción glandular, sequedad de boca, sed, dificultad para deglutir y hablar, dilatación pupilar, taquicardia, en ocasiones hipertensión, enrojecimiento de la piel por vasodilatación cutánea y disminución de la sudoración y alteración en el musculo liso del sistema gastrointestinal e hipertermia (Repetto \& Repetto, 2009).

En cuanto a los síntomas centrales, según Salcedo y Martínez (2009), al tener la propiedad la escopolamina de cruzar la barrera hematoencefálica, se bloquean funciones colinérgicas en el sistema límbico relacionados con aprendizaje y memoria, con manifestaciones comunes como la amnesia de los eventos sucedidos después de la ingesta, estados de pasividad con actitud de «automatismo» desapareciendo los actos inteligentes de la voluntad. En algunas personas puede causar desorientación, excitación psicomotriz, alucinaciones, delirio, agre- 
sividad, convulsiones, estado de coma y en algunos casos puede causar la muerte (Álvarez, 2008).

Los anteriores reportes son generales, evidenciando que la escopolamina actúa sobre las funciones mnésicas y el comportamiento pero no se sabe exactamente cual es su acción. Sin embargo, se han realizado algunas hipótesis.

Para Uribe et ál. (2005), la intoxicación por escopolamina afecta principalmente la memoria, en gran medida porque esta depende de la acetilcolina como neurotransmisor y muy especialmente sobre los receptores colinérgicos muscarínicos del núcleo basal de Meynert en la base del lóbulo frontal. Al afectarse dicho núcleo por la acción anticolinérgica de la escopolamina, se altera de manera significativa la transmisión de la información a los sitios de almacenamiento, en especial al hipocampo, es decir que la información no se puede almacenar adecuadamente. En ocasiones se ha visto que el bloqueo en el núcleo no se da de forma completa, quedando algunos recuerdos inconexos de episodios que se vivieron en el periodo de la intoxicación y estos suelen aparecer como destellos.

Por su parte, Bubser, Byun, Wood y Jones (2012); Ortiz, (2011) y Voss, Thienel, Reske, Ute y Kircher (2010) afirman que existe la probabilidad de que sea el bloqueo colinérgico sobre otras áreas del lóbulo frontal, lo que explique la aparición de síntomas como apatía, disminución de la ansiedad y la agresividad.

Voss et ál. (2010) y Uzcátegui (2005), han sugerido que la escopolamina puede bloquear la acción de otros neurotransmisores como la serotonina, especialmente en el sistema límbico. Este efecto podría explicar los cuadros psiquiátricos que se presentan en algunas personas, especialmente cuando existe algún tipo de alteración en la personalidad premórbida.

Los estudios han de continuar al respecto, pero hay además que considerar que en muchos de los casos de intoxicación por escopolamina, esta es combinada con otros depresores del sistema nervioso, como lo son las fenotiazinas y las benzodiacepinas. Las fenotizinas, debido a su efecto gabaérgico, ayudan a la potencialización del efecto depresor de la escopolamina e inducen somnolencia, y su efecto ansiolítico tranquiliza a la víctima. Las benzodiacepinas por su parte producen un efecto anticolinérgico sobre el núcleo basal de Meynert haciendo más evidente la amnesia en el paciente con intoxicación. Las fenotiazinas potencian de alguna manera el efecto depresor, que dado el efecto antidopaminérgico facilita la inhibición de la agresividad, posiblemente debido a la acción que ejerce sobre el sistema límbico (Uribe et ál., 2005).

\section{Implicaciones neuropsicológicas}

$\mathrm{Al}$ producirse cambios a nivel neurológico como consecuencia de la intoxicación por escopolamina, paralelamente se pueden producir alteraciones a nivel de funciones superiores. La transmision colinérgica relacionada con los procesos cognitivos se discute desde Deutsch (1971) quien formuló su hipótesis acerca de los cambios de sensibilidad postsináptica subyacentes al aprendizaje y memoria, de esta manera afirmó que se presentan alteraciones en el almacenamiento de la nueva información, en la memoria, la atención sostenida, la vigilancia y el arousal. Desde entonces estudios han demostrado que la memoria, el aprendizaje, la velocidad de procesamiento y la atención, son modulados por cambios en la vía colinergica (Voss et ál., 2010).

Ardila, et ál. (2006) señalaron similitudes entre la intoxicación por escopolamina y la amnesia global transitoria. En ambas se encuentra un déficit abrupto en la memoria de los eventos recientes (amnesia anterógrada). Sin embargo, durante el episodio amnésico, el sujeto mantiene una identidad personal y puede ejecutar adecuadamente sus actividades normales. En relación con las funciones ejecutivas los autores enfatizaron que el efecto de la intoxicación tiene consecuencias en toma de decisiones.

En cuanto a la cognición social, Kamboj y Curran (2006) sugieren que la hipofunción colinérgica inducida afecta la precisión en el reconocimiento de expresiones faciales de disgusto y enojo. Esto podría explicarse según lo dicho por Tirapu, Pérez, Erekatxo y Pelegrín (2007) quienes afirman que la teoría de la mente se refiere a la habilidad para comprender y predecir la conducta de otras personas, sus conocimientos, sus emociones, sus intenciones y sus creencias. Según Adolphs (2002), el reconocimiento de expresiones, se relaciona directamente con el hecho de que el sujeto realice un proceso perceptivo, el cual pueda activar la ejecución 
de tareas que requieren hacer un juicio de reconocimiento únicamente a partir de propiedades físicas del estímulo. Por el contrario, el reconocimiento, requiere de información adicional que no está disponible en los estímulos que se perciben directamente y por consiguiente, requiere de algún tipo de memoria.

El reconocimiento de emociones desde las expresiones faciales, requiere de conocimiento adicional sobre las contingencias entre las expresiones y muchos otros estímulos que se han asociado directa o indirectamente con la situación. Por ejemplo, el contexto donde se observa el rostro, la posición en el espacio del mismo, información adicional sobre la persona con ese rostro, entre otros aspectos, ninguno de los cuales pueden tomarse aislados del rostro (Adolphs, 2002).

Lo anterior habilita al individuo para actuar sin dificultades en sus relaciones interpersonales, ya sean íntimas o profesionales, pero aún más importante le permite anticipar o predecir, establecer relaciones afectivas, indispensables en el proceso de socialización (Téllez, 2006). De esta manera cuando este proceso falla, se ven afectados la percepción, el procesamiento y la evaluación de los estímulos que permiten tener una representación del entorno social (Tirapu et ál., 2007).

Las alteraciones en cognición social se relacionan con el efecto que produce la escopolamina al bloquear la vía colinérgica en áreas del lóbulo frontal y que están directamente implicadas con procesos que se activan en situaciones de interacción social, los cuales permiten percibir, evaluar y responder ante dichas situaciones, no solo valorando las propias impresiones de la persona sino infiriendo las de los demás, y estos procesos permiten la interacción satisfactoria del individuo con el medio, por lo cual su déficits resultan en interacciones inadecuadas, respuestas inapropiadas y aislamiento social.

En la revisión teórica también se evidencia que la mezcla de escopolamina con benzodiacepinas, puede producir amnesia anterograda (Kamboj \& Curran, 2006).

Finalmente lo reportado por la literatura muestra que las funciones cognitivas que se han visto afectadas en mayor medida son: el aprendizaje a través de listas de palabras, memoria tanto a nivel semántico como episódico así como también el registro de información, recuperación léxica, evocación libre, velocidad del procesamiento de información, atención, funciones visoespaciales y funciones ejecutivas (Ebert \& Kirch, 1998; Kamboj \& Curran, 2006; Seo, 2009).

\section{Implicaciones psiquiátricas}

La intoxicación por escopolamina, como se mencionó anteriormente, puede generar síntomas como alucinaciones, desorientación, agitación psicomotora, ideas delirantes, agresividad o alteraciones en el estado de ánimo que son fácilmente confundibles con cuadros psiquiátricos. El diagnóstico diferencial dependerá especialmente de los resultados de los exámenes toxicológicos y del acompañamiento de los síntomas periféricos.

Pero llegar al punto de establecer si los síntomas dependen o no de la intoxicación por escopolamina, más aun cuando ya ha pasado el episodio agudo de intoxicación, no es fácil.

Las revisiones realizadas por Wiebe, Sigurdson y Katz (2008) y por Salcedo y Martínez (2009) sobre la intoxicación por escopolamina, indican que los síntomas suelen aparecer de una a cuatro horas después de su consumo (algunas veces puede retrasarse hasta 12 horas) y con un manejo intrahospitalario pueden remitir alrededor de dos días después por el vaciamiento gástrico, por ello en el trascurso del estudio de los pacientes pueden generarse diagnósticos como episodio psicótico agudo o episodio disociativo.

Además de esto, desde la psiquiatría también se ha cuestionado sobre la aparición de enfermedades mentales posterior a la intoxicación con escopolamina, a pesar de eso durante la revisión no se encontraron estudios específicos del tema pero se retoman aspectos que tienen en cuenta el consumo de sustancias psicoactivas y el inicio de enfermedad mental encontrando por la publicación realizada por Halgin y Krauss (2003), por Gutiérrez (2000) y por Gutiérrez et ál. (2000) en donde se plantea que existe una asociación de 43.2\% entre consumo de psicotóxicos y cuadro psicótico de primera vez.

Lo anterior brinda un panorama general de la facilidad con la que se puede confundir un cuadro de intoxicación por escopolamina y un trastorno mental, 
aspectos que más adelante se debe tener en cuenta para el análisis del presente caso.

Finalmente, se hace mención que muchos de los estudios realizados con escopolamina, han sido de corte experimental en modelos animales prevalentemente (v. g. Acosta et ál., 2009; Furey, Pietrini, Haxby \& Drevets, 2008; Klinkenberg \& Blokland, 2011; Perez, Martínez, Hernandez \& Rojas, 1998; Yamamoto et ál., 2011) y hay prácticamente inexistencia de reportes de casos clínicos (v. g. Bedard, Lemay, Gagnon, Massonb \& Paquet, 1999; Little, Johnson, Minichiello, Weingartner \& Sunderland, 1998; Mintzer, Kleykamp \& Griffiths, 2010; Obonsawin et ál., 1998; Parrott, 1998), donde en todos ellos se trabajó en condiciones controladas en voluntarios, los efectos de la escopolamina en relación con otras sustancias en tareas cognitivas específicas. Solo en un estudio realizado por Vallersnes, Lund, Duns, Netland y Rasmussen (2009) investigaron sobre las intoxicaciones agudas en Oslo en el año 2008 debido a una epidemia de envenenamiento entre los usuarios de drogas ilícitas causadas por falsas pastillas Rohypnol, las cuales contenían escopolamina. Dentro de los resultados encontraron 44 casos de probable intoxicación por escopolamina. Las principales características clínicas fueron midriasis, alucinaciones visuales, comportamiento agresivo, agitación y coma. Encontraron que los pacientes no tenían un diagnóstico clínico de síndrome anticolinérgico, el diagnóstico más frecuente hasta este punto es especificado psicosis inducida por drogas. Diez pacientes fueron ingresados en hospitales psiquiátricos, el resto se recuperó en las unidades médicas. Finalmente concluyeron que la intoxicación por escopolamina es rara, pero describieron el síndrome anticolinérgico resultante. El síndrome no era reconocido hasta el resultado del análisis forense mientras tanto los pacientes fueron diagnosticados y manejados de forma errónea.

Lo anterior muestra que aunque se han realizado algunos estudios sobre la escopolamina y sus efectos a nivel del sistema nervioso central, aun es un área poco explorada y son muchos los cuestionamientos que pueden surgir alrededor, por lo anterior el objetivo del presente estudio es identificar las implicaciones neuropsicológicas, neurológicas y psiquiátricas en un caso de intoxicación por escopolamina, lo que servirá como evidencia para el diseño de programas que ayuden a disminuir las secuelas o los posibles efectos que genera esta intoxicación.

\section{Método}

\section{Diseño}

Estudio de caso el cual de acuerdo con Hernández, Fernández y Baptista (2006) es una metodología que utiliza los procesos de investigación cualitativa, cuantitativa o mixta; analizan a nivel profundo una unidad para responder al planteamiento de un problema, probar hipótesis y desarrollar alguna teoría.

\section{Sujeto}

Paciente de sexo masculino de 43 años de edad, con escolaridad de octavo grado y ocupación conductor, quien sufrió intoxicación por escopolamina aproximadamente cinco meses antes de la valoración. Posterior al episodio de intoxicación permaneció fuera de la casa por cinco días, al encontrarlo presentaba fallas en memoria, no reconocía a sus familiares y tenía pérdida del control de esfínteres. Fue trasladado a un hospital donde requirió manejo en UCI con intubación por falla cardiaca. Posteriormente retornó a su empleo durante un mes con dos episodios más de pérdidas de su hogar, el primero durante dos días (fin de semana) y el segundo episodio dura nueve días donde fue encontrado en actitud perpleja y fue llevado a consulta médica ambulatoria. Tres meses después de superar el episodio agudo de intoxicación sale de su casa y permanece perdido durante un mes, es encontrado por sus familiares en otra ciudad, en condición de indigencia, desorientación, no reconocimiento de sus familiares, síntomas psicóticos y no recuerdo de lo sucedido durante ese periodo de tiempo. Por lo cual es llevado a una clínica psiquiátrica a donde ingresa con un diagnóstico de trastorno disociativo. Le toman una TAC cerebral con resultados normales y en pruebas de toxicología los resultados fueron negativos. Al regresar es remitido al área de neuropsicología para iniciar proceso de evaluación. Durante la misma el paciente refirió fallas subjetivas como labilidad emocional, dificultades atencionales y fallas mnesicas «se me ha olvidado todo, hasta las cosas de cuando era pequeño». 


\section{Instrumentos}

Escala de inteligencia de Weschler Wais III: proporciona una medida general de la función intelectual en adolescentes mayores y adultos. La batería compuesta por 11 subpruebas principales y tres complementarias. Se evalúa nivel global (CI Total), Nivel verbal (CI Verbal) y nivel de ejecución (CI ejecutivo). En cuanto a la validez se señala que existe una correlación sustancial (.80 y superior) entre el WAIS-III y su predecesor (Strauss, Sherman \& Spreen, 2006).

Prueba de ejecución continua: la cual permite analizar la atención sostenida en tareas que requieren de un trabajo continuo que el sujeto ha de realizar de manera monótona sin detenerse, con el riesgo que se dé un detrimento o menoscabo de la atención. Fiabilidad de .83 (Narbona \& Chevrie-Muller, 1997).

Trail Making Test (TMT A y TMT B): Se considera que la parte A mide habilidades motoras, viso-espaciales de búsqueda visual y atención sostenida; y la parte B implica además flexibilidad mental y atención dividida. La confiabilidad del TMT-A está entre .76 y .89 y la del TMT-B entre .86 y .94 (Rabin, Barr \& Burton, 2005).

Test Verbal de California: evalúa el proceso de aprendizaje verbal y la cantidad de información que se puede adquirir y retener utilizando (memoria verbal) usando una tarea de lista-aprendizaje de múltiple-ensayo. Se emplea en personas entre los 17 y los 80 años de edad y su fiabilidad original ha sido de .74 (Strauss et ál., 2006).

Figura de Rey-Osterreith: evalúa habilidades visoespaciales, construccionales y la memoria visual. Incluye además destrezas de planeación y organización y estrategias de resolución de problemas tanto en las funciones preceptuales, motoras y de memoria. En general, los datos de los estudios analíticos y el factor de correlación apoyan la validez de la Figura de Rey como una medida que incluye la capacidad visuoconstructiva (copia) y la memoria (recuerdo y reconocimiento). Se espera en población normal una diferencia de hasta 10 puntos entre la copia y el recuerdo. La sensibilidad está probada entre .61 y .84 (Rey \& Osterrieth, 1993).

Token Test: su objetivo es evaluar la comprensión de instrucciones verbales de complejidad creciente. No se hacen correcciones por edad ni por escolaridad. Fue inicialmente desarrollado por Vignolo y luego fueron apareciendo otras versiones en otros colores y menor núemro de ítems. En el estudio se empleó la de 39 instrucciones. Se han encontrado altas correlaciones con otras pruebas como el PPVT de .71 (Strauss et ál., 2006).

Test de fluidez verbal semántica y fonológica: la fluidez verbal se mide usualmente por el número de palabras producidas en un minuto en una categoría dada. Las dos principales categorías se refieren a la semántica (palabras que pertenecen a determinada categoría: animales o frutas). La fluidez fonológica implica la evocación de palabras que empiezan por un fonema particular, generalmente F, A y S, exceptuando nombres propios durante un tiempo determinado (un minuto). El examinador contabiliza el tiempo y anota las palabras generadas, incluso las erróneas. Después de un minuto, se culmina la prueba. Confiabilidad test- retest ed de .88 (Ardila \& Rosselli, 1992).

Matrices de Raven: consta de 60 matrices o dibujos, a cada uno de estos dibujos le falta una parte. Estas matrices se agrupan en cinco series (A, B, C, D, E), cada serie está integrada por 12 ítems. Dentro de cada serie, a medida que avanza la tarea, va en aumento el grado de complejidad de la misma. Siempre la serie precedente presenta menor complejidad que la siguiente, aunque en cada nueva serie los primeros ítems tienen menor complejidad que los últimos que le antecedieron de la serie anterior. Valores de fiabilidad .70 a .86 (González, 2007).

Behavior Rating Inventory of Executive Function: inventario de conducta frontal que permite establecer en sus ocho dominios los cambios conductuales y cognoscitivos específicos derivados del daño en el lóbulo frontal. El índice de regulación de conducta tiene una confiabilidad de .66 a .95 y para el índice metacognitivo de .74 a .98 (Strauss et ál., 2006).

Test de fluidez de diseños (RUFF): Es una prueba de fluidez no verbal, análoga a la prueba (FAS), la prueba está dividida en cinco tareas diferentes: son 5 hojas, cada una se encuentra dividida en 35 cuadrados y en cada uno de ellos se encuentran cinco puntos; el objetivo es dibujar tantos diseños únicos como sea posible en un intervalo de un minuto; esta prueba es sensible en cuanto a edad y nivel educativo del sujeto evaluado. La fiabilidad original del instrumento es .91 (Strauss et ál., 2006).

Test de clasificación de tarjetas de Wisconsin: evalúa la capacidad para generar criterios de clasificación, pero 
sobre todo la capacidad para cambiar de criterio de clasificación (flexibilidad) con base en cambios repentinos en las condiciones de la prueba. Resultados de fiabilidad se han dado en diferentes países y fluctúan en .49 y .83 (Strauss et ál., 2006).

Test de las miradas: evalúa la habilidad de reconocer el estado mental de una persona a través de la lectura de la expresión de la mirada. Se desarrolló sobre la base de los conceptos de Teoría de la Mente que consideran que los sujetos sanos son capaces de determinar el estado mental de una persona a partir de la expresión de la mirada (Román et ál., 2012).

Dilemas sociales personales e impersonales: evalúa la empatía y el juicio moral a través del análisis de unas situaciones particulares que pretenden generar conductas prosociales en el evaluado (Tirapu et ál., 2007).

Además se amplió información con la prueba de personalidad MMPI, el cual es un instrumento para la evaluación de las características psicopatológicas globales y específicas del individuo, trastornos psicosomáticos, intereses y personalidad psicopatológica. La prueba consta de 338 ítems que cubren un total de 50 escalas. Tres de estas escalas son de segundo orden y agrupan todas las anteriores, como son las de alteraciones emocionales o internalizadas, alteraciones del pensamiento y alteraciones del comportamiento o externalizadas.

\section{Procedimiento}

Fase 1. A la consulta externa de una clínica psiquiátrica, luego de una estancia hospitalaria de un mes, es remitido a valoración por neuropsicología el paciente del cual se constituye el presente caso clínico y dada la relevancia de la temática y la interacción clínica de factores neuropsicológicos, neurológicos y psiquiátricos, se procedió a obtener el consentimiento informado del paciente y posteriormente se realizó el proceso de anamnesis

Fase 2. Se realizó la evaluación neuropsicológica por medio de la aplicación de las diferentes pruebas neuropsicológicas mencionadas anteriormente. Adicionalmente se aplicó el MMPI con el fin de evaluar algunas variables de personalidad.

Fase 3. Análisis de resultados a nivel cuantitativo y cualitativo.

\section{Consideraciones éticas}

Para el presente trabajo se tuvieron en cuenta las consideraciones éticas de la Ley 1090 de 2006 del Congreso de la República por la cual se reglamenta el ejercicio de la profesión de psicología, se dicta el código deontológico y bioético y otras disposiciones (Congreso de la república de Colombia, 2006).

\section{Resultados}

\section{Resultados cuantitativos}

A continuación se presentan los resultados cuantitativos de la evaluación realizada. En la tabla 1 se presentan los resultados obtenidos en las subescalas de la prueba de inteligencia WAIS. En la tabla 2 se muestran los resultados de la evaluación neuropsicológica realizada discriminando la función cognitiva evaluada.

Tabla 1. Puntuaciones subescalas WAIS

\begin{tabular}{llll}
\hline \hline Escalas verbales & CI & Escalas manipulativas & CI \\
\hline Información & 6 & Clave de números & 8 \\
Comprensión & 12 & Figuras incompletas & 5 \\
Aritmética & 8 & Cubos & 8 \\
Semejanzas & 12 & Historietas & 5 \\
Dígitos & 5 & Rompecabezas & 10 \\
Vocabulario & 7 & CI manipulativo & 81 \\
CI verbal & 85 & & \\
CI total: 83 & & & \\
\hline \hline
\end{tabular}


Tabla 2. Resultados cuantitativos de la evaluación neuropsicológica

\begin{tabular}{|c|c|c|c|}
\hline Función cognitiva & \multicolumn{2}{|c|}{ Prueba aplicada } & Puntaje \\
\hline & \multicolumn{2}{|c|}{ Ejecución continua } & $\begin{array}{l}\text { Auditiva: Pd: 8/16 E: } 1 \\
\text { Visual: T: 50» Pd: 16/16 }\end{array}$ \\
\hline & \multicolumn{2}{|c|}{ Dígitos directos } & Pd: 4/7+2 \\
\hline & \multicolumn{2}{|c|}{ Dígitos inversos } & Pd: $3 / 7+2$ \\
\hline & \multirow{2}{*}{ TMT } & Parte A & T: 175»Pd: 24/24 \\
\hline & & Parte B & T: 134»Pd: 24/24 \\
\hline \multirow{7}{*}{ Atención y memoria } & \multirow{6}{*}{ California } & Retención inmediata & Pd: 5/16 \\
\hline & & $\%$ de aprendizaje & $43.75 \%$ \\
\hline & & Recuerdo 5 minutos & Pd: 6/13 \\
\hline & & Recuerdo 20 minutos & Pd: 6/13 \\
\hline & & $\begin{array}{l}\text { Reconocimiento/falsos } \\
\text { positivos }\end{array}$ & Pd: 10 FP: 16 \\
\hline & & Fenómenos patológicos & I: 7 P: 6 \\
\hline & \multicolumn{2}{|c|}{ Figura de Rey - Recuerdo } & Pd: 9.5/27.5 \\
\hline & \multicolumn{2}{|c|}{ Comprensión material verbal complejo } & Pd: $8 / 9$ \\
\hline \multirow[t]{2}{*}{ Lenguaje } & \multicolumn{2}{|l|}{ Token test } & Pd: $140 / 163$ \\
\hline & \multicolumn{2}{|c|}{ Fluidez verbal semántica } & M: 12 \\
\hline Praxias & \multicolumn{2}{|c|}{ Figura de Rey } & Pd: $27.5 / 36$ \\
\hline \multirow{7}{*}{ Función ejecutiva } & \multicolumn{2}{|l|}{ Semejanzas } & Pd: $16 / 38$ \\
\hline & \multicolumn{2}{|l|}{ Aritmética } & Pd: 7/16 \\
\hline & \multicolumn{2}{|c|}{ Fluidez verbal fonológica } & M: 7 \\
\hline & \multicolumn{2}{|c|}{ Matrices de Raven } & 79 \\
\hline & \multicolumn{2}{|l|}{ Ruff } & $\begin{array}{l}\text { Diseños únicos: Pd:76 } \\
\text { Perseveraciones: Pd:9 }\end{array}$ \\
\hline & \multirow{2}{*}{ Wisconsin } & $\%$ errores perseverativos & $17.64 \%$ \\
\hline & & \% conceptualización & $61.76 \%$ \\
\hline
\end{tabular}

PD: Puntuación directa. T: Tiempo. M: Media E: Errores. FP: Falsos positivos. I: intrusiones P: Perseveraciones

\section{Resultados cualitativos}

Inteligencia: nivel de inteligencia dentro de la media, con puntuaciones similares en las escalas verbales y manipulativas y sin evidencia de alteraciones en ninguno de los subcomponentes intelectuales evaluados de manera estadísticamente significativa de acuerdo a las pruebas aplicadas.

Procesamiento cognoscitivo: se destaca un perfil cognoscitivo global dentro de los límites normales, con evidencia de fallas específicas a nivel de los procesos atencionales, nuevos aprendizajes, comprensión en relación con la complejidad y funciones ejecutivas. Específicamente por áreas se encuentra:

Atención: baja velocidad de procesamiento de la información, dificultades para mantenerse centrado durante los tiempos requeridos en tareas de atención auditiva, con alto número de errores tipo comisión en la primera parte y omisiones asociadas a rápida fatiga. 
En atención visual se destaca ausencia de errores, pero con evidencia de tiempos sustancialmente superiores a los requeridos para completar las tareas.

Memoria: nuevos aprendizajes verbales caracterizados por nivel de retención inmediata en el límite normal bajo, con dificultades para lograr un efectivo almacenamiento de la información suministrada en los diferentes ensayos, pérdida evocativa en el corto plazo y la proporción de claves semánticas no le fue de mayor ayuda. En memoria visual hay evidencia de pérdida de elementos de detalle, con tendencia a mantener en general, el marco contenedor de la información.

Lenguaje: en relación con la complejidad de las instrucciones verbales, se le dificulta hacer un efectivo seguimiento, aspecto también corroborado en las demás tareas, donde era necesario hacerle repetición de las instrucciones, en la medida en que estas eran más largas o complejas.

Praxias: es llamativo encontrar dificultades que se relacionan en parte a la pobre planeación de la figura, pero adicionalmente, se destaca tendencia a presentar alto número de distorsiones y desplazamientos de los elementos y el tiempo tan elevado para la copia $\left(6^{\prime} 32 »\right)$.

Funciones ejecutivas: presenta errores a nivel de la planeación de las ejecuciones, tendiendo a predominar algunas respuestas impulsivas. Emplea tiempos mayores para resolver tareas de solución de problemas y se hace llamativo que requiere mayores análisis para lograr dar una respuesta.

En el test de las miradas se evidencia tendencia a relacionar las miradas con emociones negativas, como tristeza, miedo, intranquilidad, preocupación, entre otras.

En cuanto a dilemas sociales y personales, se evidencia un nivel adecuado de comprensión de las consecuencias emocionales que tiene una circunstancia o comportamiento propio generando un estado emocional correspondiente con el supuestamente experimentado por la otra persona. Adicionalmente, en el BRIEF muestra alteración en regulación emocional

Por otra parte, los resultados de la prueba de personalidad MMPI se muestran en la figura 1.
PERFIL

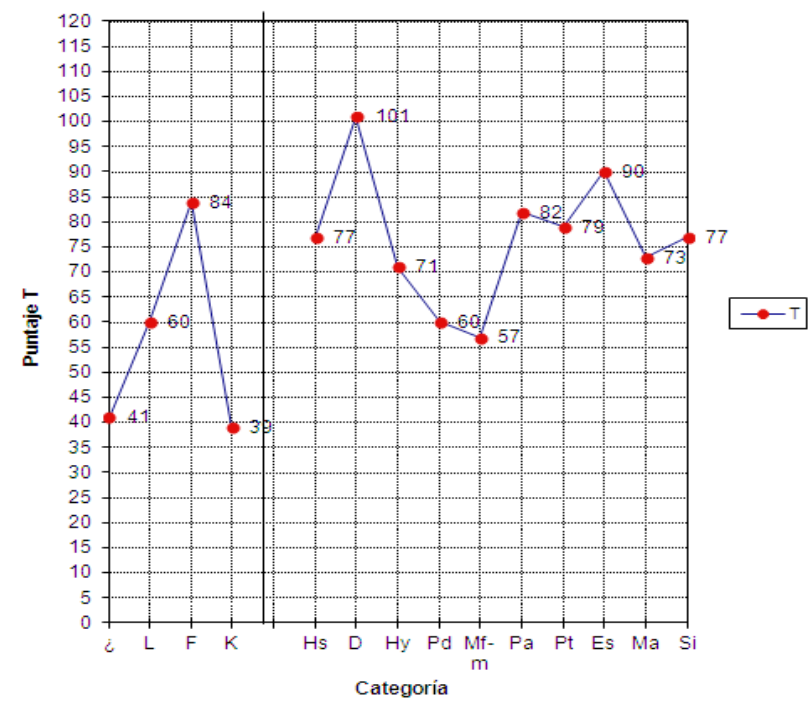

Figura 1. Perfil de la prueba de personalidad MMPI

Los resultados registrados en la gráfica indican una necesidad de parte del paciente de mostrarse de forma favorable, tiende a magnificar sus síntomas en torno a la necesidad de atención por lo que su perfil debe ser analizado con precaución. A pesar de eso, se destacan algunas características a lo largo de sus resultados como lo son tendencia al aislamiento, alto nivel de autocrítica y autodevaluación, inadecuado concepto de sí mismo, con baja tolerancia a la frustración, déficit en solución de problemas que genera que pueda magnificar sus preocupaciones y tienda a generar afecto triste.

\section{Discusión}

De acuerdo a la revisión teórica que se realizó en el presente estudio se evidencian algunas coincidencias frente a los procesos cognoscitivos que se describen como alterados en la intoxicación por escopolamina como se presenta a continuación:

El paciente presenta algunas fallas en cuanto respecta a procesos atencionales. Se observó baja velocidad de procesamiento y dificultades en el mantenimiento de la atención, especialmente en tareas de atención auditiva, presentando un gran número de errores por comisión y omisión. Esto se relaciona con estudios anteriores realizados con el fin de observar los efectos de la escopolamina en los procesos cognitivos de las personas afectadas, 
especialmente en tareas que requieren un tiempo determinado de reacción, seguimiento de objetivos, atención sostenida y memoria como un aspecto fundamental en procesos de nuevos aprendizajes (Parott, 1998).

También es importante tener en cuenta que el paciente objeto de estudio, presenta fallas a nivel de memoria, en cuanto a la realización de nuevos aprendizajes verbales caracterizados por un bajo desempeño en tareas de retención inmediata y dificultad para lograr un efectivo almacenamiento de la información suministrada en diferentes ensayos. De la misma manera presenta pérdida evocativa a corto plazo donde el suministro de claves semánticas no le es de ayuda. Lo anterior se sustenta en los hallazgos de Klinkenberg y Blokland (2011) cuando a través de su estudio para evaluar los efectos del biperideno con relación a los de la escopolamina como un modelo farmacológico para déficits colinérgicos de la memoria en ratas, afirman que la escopolamina genera cambios de comportamiento incluyendo cambios en la respuesta sensoriomotora, en la motivación, la atención y la memoria a corto plazo mientras que el biperideno por su parte, induce déficits más selectivos en la respuesta sensoriomotora y en memoria a corto plazo sin llegar a afectar la atención ni el área motivacional.

Con base en lo anterior, Mintzer et ál. (2010) por medio de su estudio para comparar los efectos de diferentes dosis de triazolam y escopolamina en aspectos que tienen que ver con la metamemoria, encontraron que los dos ejercen efectos similares, pues tanto el Triazolam como la escopolamina producen un deterioro significativo de memoria episódica pero no en lo que respecta a memoria semántica. Según estos investigadores ambas drogas producen, problemas de control y sensibilidad.

En memoria visual hay evidencia de pérdida de elementos de detalle, con tendencia a mantener en general, el marco contenedor de la información. Castañón, Martínez, López, Gómez y García (2000) y Parott (1998) afirman que la exposición a la escopolamina transdérmica como consecuencia puede presentar una marcada disminución de agudeza visual y visión borrosa grave. Esto podría explicar de alguna manera un bajo desempeño en tareas de memoria visual, lo cual es evidente en el paciente cuando se tienen en cuenta los tiempos prolongados empleados para este tipo de tareas.
Desde el punto de vista neurológico se dice que las alteraciones a nivel cognitivo que se presentan a partir de la intoxicación por escopolamina no son muy estudiadas hasta el momento pero existe la posibilidad de que la alteración de la memoria se deba a que según Uribe et ál. (2005), la escopolamina actúa sobre la acetilcolina, especialmente sobre los receptores colinérgicos muscarínicos del núcleo basal de Meynert en la base del lóbulo frontal. Al dañarse este núcleo se ve alterada la transmisión de la información a los sitios de almacenamiento, en especial al hipocampo.

De acuerdo a lo anterior, también es importante tener en cuenta a Voss et ál. (2010), cuando afirman que es el antagonismo de receptores muscarínicos quien da lugar a deficiencias cognitivas específicas, predominantemente en cuanto a tareas cuyo rendimiento implican memoria.

En cuanto a lenguaje el paciente presenta dificultades que se destacan en relación con la complejidad de las instrucciones verbales, le cuesta trabajo hacer un efectivo seguimiento, aspecto también corroborado en las demás tareas, donde era necesario hacerle repetición de las instrucciones, en la medida en que estas eran más largas o complejas. Esto se puede explicar a través de las dificultades que presenta a nivel de atención y velocidad de procesamiento, pues al no haber una adecuada asimilación de la información se hace necesaria la constante repetición de instrucción y se presenta prolongación de los tiempos requeridos para la ejecución de la tarea.

En Práxias se encontró que el paciente presenta dificultades que se relacionan con una pobre planeación de la figura de Rey, destacándose tendencia a presentar alto número de distorsiones y desplazamientos de los elementos. El tiempo utilizado para la copia de la figura de rey fue muy superior al esperado. Teniendo en cuenta que el paciente presenta dificultades a nivel de atención es esperable que evidencie problemas en planeación y debido a su dificultad en cuanto a velocidad de procesamiento es entendible la prolongación del tiempo empleado para la copia de la figura.

En cuanto a funciones ejecutivas se observó que el paciente presenta errores a nivel de la planeación, impulsividad o bajo control inhibitorio y tiempos prolongados en resolución de problemas. Esto se puede explicar desde la teoría de Pueyo (2001) quien afirma que el bajo 
control inhibitorio está influenciado por la baja tolerancia a la frustración y pobres habilidades en la planeación para llegar a la resolución efectiva de un problema, esto de alguna marera podría explicar el desempeño del paciente en tareas que involucran este tipo de habilidades.

Se evidenciaron alteraciones en el foco atencional, durante el proceso de evaluación, el paciente logra dirigir la atención hacia los estímulos requeridos pero no la mantiene durante periodos prolongados cuando la información se presenta por canal auditivo, evidenciando una pronta fatiga, lo que también se relaciona con el componente de esfuerzo, que sumado a lo anterior, genera que el paciente requiera de altos tiempos para la ejecución de la tarea aunque se mantenga alerta.

El componente de memoria, también se ve alterado secundario posiblemente a los fallos atencionales puesto que posterior al proceso de atención se debe de dar una adecuada consolidación de la información presentada.

Finalmente en cuanto al componente de acción no se evidencian fallos en la automonitorización aunque sí se presentan dificultades en cognición social y empatía, evaluado por medio de el test de las miradas, lo que puede estar directamente relacionado con lo dicho por Kamboj y Curran (2006) y Kiyofumi y Toshitaka (2003) quienes sugieren que la hipofunción colinérgica inducida afecta la precisión en el reconocimiento de expresiones faciales de disgusto y enojo.

Con base en lo mencionado anteriormente y de acuerdo con Téllez (2006), se podría asumir que las fallas que se evidencian en el paciente en cuanto respecta a cognición social pueden estar interfiriendo en sus habilidades para interpretar los estados mentales y emocionales tanto propios como ajenos, lo cual se considera la base de otros fenómenos psicológicos como la empatía, la capacidad de autoconocimiento, las relaciones interpersonales y otros déficits en las aptitudes sociales.

De acuerdo con lo anterior este autor sugiere, que las personas presentan una habilidad automática para atribuir deseos, intenciones, emociones, estados de conocimiento o pensamientos a otras personas y darse cuenta de que en muchas ocasiones son distintas a las propias (teoría de la mente). Esto conlleva a la representación interna de los estados mentales de las otras personas, al fallar esta capacidad se presentan dificultades a nivel de empatía y por ende en las relaciones sociales o interpersonales de los sujetos. Estas dificultades a su vez pueden llevar a que las personas tengan problemas a la hora de anticipar lo que los demás pueden pensar sobre su comportamiento así como para idear una mentira o para comprender engaños. Lo anterior además de la posible mezcla de la burundanga con otros depresores del SNC está relacionada con la dificultad de identificar situaciones de amenaza.

Frente al área de psiquiatría es importante destacar la repetición de eventos que presenta el paciente de desorientación, fallas mnesicas y síntomas psicóticos, lo que no es descrito en la revisión realizada teniendo en cuenta el tiempo transcurrido entre el evento de intoxicación y el último episodio de pérdida. Lo anterior permite pensar que, como describen Gutiérrez et ál. (2000), el episodio de intoxicación pudo haber disparado la aparición de un cuadro mental.

Llama la atención el perfil obtenido mediante la prueba de personalidad, el cual, a pesar de la necesidad de mantener precaución en su interpretación, puede sugerir unos rasgos de personalidad del cluster A, específicamente de personalidad esquizoide en donde existe una tendencia a disfrutar poco de las relaciones interpersonales, escoge actividades solitarias, tiene poco interés en tener experiencias sexuales, no tiene amigos íntimos y mantiene un aplanamiento afectivo que en ocasiones varía con episodios depresivos como lo describió la familiar del paciente (American Psychiatric Association, 2002).

Lo anterior también puede explicar la aparición de los síntomas psicóticos por la intoxicación, puesto que a pesar de que la mayoría de personas con rasgos de personalidad esquizoides no presentan delirios ni alucinaciones, si tienen más vulnerabilidad a sufrirlos, y como se planteó en el estudio ya citado realizado por Gutiérrez et ál. (2000), así como el realizado por Atri et ál. (2004), estos pueden ser fácilmente desencadenados posterior a la inducción de sustancias psicoactivas en el organismo, más aun cuando hay factores predisponentes para el mismo.

Otro aspecto a resaltar son las quejas subjetivas del paciente, en donde manifiesta que las fallas de memoria se han tornado globales posterior al evento estresante, aspecto que tampoco ha sido descrito en ninguno de los estudios revisados. A pesar de eso la evaluación neurop- 
sicológica permite evidenciar una memoria a largo plazo dentro de un límite normal bajo. Esto se puede explicar además por los resultados de la prueba de personalidad MMPI en donde se registra una tendencia del paciente a magnificar síntomas en busca de una mayor atención.

Es importante cuestionar la importancia de los antecedentes familiares dentro del posible diagnóstico del paciente.

A partir de los hallazgos encontrados en la evaluación neuropsicológica y la bibliografía existente se puede concluir que aunque la escopolamina tiene efectos marcados sobre procesos cognitivos, dichos efectos dependen en gran medida de la dosis suministrada y de si esta se utiliza pura o mezclada con algún depresor del sistema nervioso central.

También es importante mencionar que los síntomas evidenciados en una intoxicación por escopolamina pueden ser confundidos con síntomas de perfiles psiquiátricos lo cual puede generar un diagnóstico erróneo, debido a esto es importante la realización de exámenes toxicológicos para descartar una intoxicación con un fármaco.

Ahora bien, en cuanto a lo revisado en la literatura sobre el perfil neuropsicológico de casos relacionados con intoxicación por escopolamina, se resalta la concordancia que existe con el caso estudiado en dificultades en velocidad de procesamiento de la información, atención sostenida, seguimiento de instrucciones, pobre regulación emocional, almacenamiento de información, evocación y planeación.

Desde el punto de vista neurológico se podría concluir que las fallas que presenta el paciente en cuanto se refiere a procesos cognitivos radican básicamente al bloqueo colinérgico que este tipo de intoxicación causa a nivel de estructuras frontales y temporales. Específicamente en núcleo de Meynert y receptores tanto muscarínicos como nicotínicos.

Desde la perspectiva de Blake et ál. (2012), la formación previa de la administración sistémica del antagonista muscarínico escopolamina conduce a la alteración de la memoria especialmente la anterógrada en pruebas de retención. La administración de la colina receptor nicotínico $\alpha 7$-agonista (Ch) en el hipocampo dorsal inmediatamente después permite la reactivación de la memoria cuando se trata de un deterioro de la memoria inducida por escopolamina. En uno de sus estudios se vio que el efecto de $\mathrm{Ch}$ es dependiente del tiempo y el rendimiento en tareas de retención no se ve afectado. Según estos autores la Ch. ejerce sus efectos de modulación en procesos de memoria y reconsolidación. El deterioro de la memoria inducida por dosis bajas de escopolamina produce un fallo en la memoria y la expresión, no es un déficit de almacenamiento. Por lo tanto, la reconsolidación, entre otras funciones, puede servir para cambiar la expresión de la memoria en las pruebas posteriores.

\section{Referencias}

Acosta, J., Mayer, L., Talboom, J., Zay, C., Scheldruo, M., Castillo, J., Demers, L., Enders, C. \& Biomonte, N. (2009). Premarin Improves Memory, Prevents Scopolamine-Induced Amnesia and Increases Number of Basal Forebrain Choline Acetyltransferase Positive Cells in Middle-Aged Surgically Menopausal Rats. Recuperado en: www.ncbi.nlm.nih.gov/pubmed/19101559

Adolphs, R. (2002). Recognizing Emotion from facial Expressions: Psychological and Neurological Mechanisms. Behavioral and Cognitive Neuroscience Reviews, 1 (1), 21-62.

Álvarez, L. (2008). Borrachero, cacao sabanero o floripondio (brugmansia spp.). Un grupo de plantas por redescubrir en la biodiversidad latinoamericana. Cultura y Droga, 13 (15), 77-93.

American Psychiatric Association (2002). DSM IV-TR Manual diagnóstico y estadístico de los trastornos mentales. España: Masson.

Ardila, A., Moreno, C. \& Gómez, S. (2006). Intoxicación por escopolamina ('burundanga'): pérdida de la capacidad de tomar decisiones. Revista de Neurología, 42 (2), 125-128.

Ardila, A. \& Rosselli, M. (1992) Neuropsicología clínica. Prensa Creativa, Medellín.

Atri, A., Norman, K., Nicolas, M., Cramer, S., Hasselmo, M., Sherman, S., Kirchhoff, B., Greicius, M., Breiter, H., \& Stern, C. (2004). Blockade of Central Cholinergic Receptors Impairs New Learning and Increases Proactive Interference in a Word Paired-Associate Memory Task. Behavioral Neuroscience, 118 (1), 223-236. 
Bédard, M., Lemay, S., Gagnon, J., Massonb, H. \& Paquet, F. (1999). Induction of a Transient Dysexecutive Syndrome in Parkinson's Disease Using A Subclinical Dose of Scopolamine. Behavioural Neurology, 11, 187-195.

Blake, M., Boccia, M., Krawczyk, M., Delorenzi, A. \& Baratti, C. (2012). Choline reverses scopolamineinduced memory impairment by improving memory reconsolidation. Neurobiology of Learning and Memory, 98 (2), 112-121.

Bubser, M., Byun, N., Wood, M. \& Jones, C. (2012). Muscarinic Receptor Pharmacology and Circuitry for the Modulation of Cognition. Handbook Experimental Pharmacology. New York: Springer.

Castañón, L., Martínez. J., López, S., Gómez, J. \& García, M. (2000). Intoxicación por Datura stramonium. Anales Españoles de Pediatría, 53 (1), 53-55.

Congreso de la República de Colombia (2006). Ley 1090 de 2006. Recuperado en: www.psicologiaprospectiva. com/introley1090.html

Córdoba, D. (2006). Toxicología. Bogotá: Manual Moderno.

Deutsch, J. (1971). The Cholinergic Synapse and the Site of Memory. Science, 174, 788-794.

Ebert, U. \& Kirch, W. (1998). Scopolamine Model of Dementia: Electroencephalogram Findings and Cognitive Performance. European Journal Clinic Investigation, 28 (11), 944-949.

Furey, M., Pietrini, P., Haxby, J. \& Drevets, W. (2008). Selective Effects of Cholinergic Modulation on Task Performance during Selective Attention. Neuropsychopharmacology, 33 (4), 913-923.

Gonzales, F. (2007). Instrumentos de evaluación psicológica. La Habana: Editorial Ciencias Médicas.

Gupta, S., Peshin, S., Srivastava, A. \& Kaleekal, T. (2003). A Study of Childhood Poisoning at National Poisons Information Centre, All India Institute of Medical Sciences, New Delhi. Journal of Occupational Health, 45, 191-196.

Gutiérrez, M. (2000). Intoxicación aguda por burundanga. Hospital San Juan de Dios. Recuperado en: www. aibarra.org/Guias/10-12.htm.

Gutiérrez, O., Gutiérrez, J., Arboleda, M., Escobar, L., Osorio, I., Londoño, M., Castrillon, J., Viteri, R. \& Motoa, C. (2000). Cuadro psicótico agudo asociación con consumo de psicotóxicos. Revista Colombiana de Psiquiatría, 29 (4), 367-374.
Halgin, R. \& Krauss, S. (2003). Psicología de la anormalidad. México: McGraw-Hill

Hernández, R., Fernández, C. \& Baptista, P. (2006). Metodología de la Investigación. Cuarta Edición. México: Mc Graw Hill.

Kamboj, S. \& Curran, H. (2006). Scopolamine Induces Impairments In The Recognition Of Human Facial Expressions of Anger And Disgust. Psychopharmacology, 185, 529-535.

Kiyofumi, Y. \& Toshitaka, N. (2003). Brain-Derived Neurotrophic Factor/TrkB Signaling in Memory Processes. Journal of Pharmacological Sciences, 91, 267-270.

Klinkenberg, I. \& Blokland, A. (2011). A Comparison of Scopolamine and Biperiden as a Rodent Model for Cholinergic Cognitive Impairment. Recuperado en: www.ncbi.nlm. nih.gov/pmc/articles/PMC3090581/

Little, J., Johnson, D., Minichiello, M., Weingartner, H. \& Sunderland, T. (1998). Combined Nicotinic and Muscarinic Blockade in Elderly Normal Volunteers: Cognitive, Behavioral, and Physiologic Responses. Neuropsychopharmacology, 1 (19), 60-69.

Mintzer, M., Kleykamp, B. \& Griffiths, R. (2010). Dose Effects of Triazolam and Scopolamine on Metamemory. Experimental Clinical Psychopharmacology, 18 (1), 17-31.

Narbona, J. \& Chevrie-Muller, C. (1997). El lenguaje del niño. Desarrollo normal, evaluación y trastornos. Barcelona: Masson

Obonsawin, M., Robertson, A., Crawford, J., Perera, C., Walker, S., Blackmore, L., Parker, D. \& Besson, J. (1998). Non-mnestic Cognitive Function in the Scopolamine Model of Alzheimer's Disease. $\mathrm{Hu}$ man Psychopharmacology, 13, 439-449.

Ortiz, J. (2011). Epidemiología de las intoxicaciones agudas en adultos atendidas en la clínica San Juan de Dios en Cartagena en el periodo de 2009-2010. Biblioteca digital repositorio institucional.

Parrott, A. (1998). Transdermal Scopolamine: Effects upon Psychological performance and visual Fuctioning at Sea. Human Psychopharmacology, 3, 119-125.

Perez, N., Martínez, C., Hernandez, M. \& Rojas, R. (1998). Estudio preclínico de un extracto acuoso de datura Candida de flore. Instituto de Ciencias Básicas y Preclínicas de Cuba, 3 (3), 23-26. 
Pueyo, A. (2001). Impulsividad y cerebro. Departamento de Personalidad-Universidad de Barcelona.

Rabin, L. A., Barr, W. B., \& Burton, L. A. (2005). Assessment Practices of Clinical Neuropsychologists in the United States and Canada: a Survey of INS, NAN, and APA Division 40 Members. Archives of Clinical Neuropsychology, 20, 33-65.

Reppeto, M. \& Repetto, G. (2009). Toxicología fundamental. España: Ediciones Díaz de Santos.

Rey, A., \& Osterrieth, P. (1993). Translations of Excerpts from Andre Rey's Psychological Examination of Traumatic Encephalopathy and P. A. Osterrieth's The Complex Figure Test. Clinical Neuropsychologist, 7, 2-21.

Román, F., Rojas, G., Román, N., Iturry, M., Blanco, R., Leis, A., Bartoloni, L., Allegri, R. \& Argencog. (2012). Baremos del Test de la mirada, en español, en adultos normales de Buenos Aires. Revista Neuropsicología Latinoamericana, 4 (3), 1-5.

Salcedo, J. \& Martínez, I. (2009). Intoxicación por escopolamina. Federación Panamericana de Asociaciones de Facultades (Escuelas) de Medicina.

Seo, S. (2009). Mental Confusion Associated with Scopolamine Patch in Elderly with Proximal LAD Occlusion. Following the Administration of Mild Cognitive Impairment (MCI). Archives of Gerontology and Geriatrics, 49 (2), 204-207.

Strauss, E., Sherman, E. \& Spreen, O. (2006). A Compendium of Neuropsychological Tests: Administration, Norms, and Commentary, Third Edition. New York: Oxford University Press.

Téllez, J. (2006). Teoría de la mente: evolución, ontogenia, neurobiología y psicopatología. Avances en Psiquiatría Biológica. 7, 6-27.
Tirapu, J., Pérez, G., Erekatxo, M. \& Pelegrín, C. (2007). ¿Qué es la teoría de la mente? Revista Española de Neurología, 44 (8), 479-489.

Uribe, G., Moreno, C., Zamora, A. \& Acosta, P. (2005). Perfil epidemiológico de la intoxicación con burundanga en la clínica Uribe Cualla S. A. de Bogotá, D. C. Revista Panamericana de Salud Pública, 21 (3), 197-202.

Uzcátegui, L. (2005). La burundanga ataca. Revista Electrónica Gente del Siglo XXI. Publicado: 28.12.2005. Recuperado el 25 de noviembre de 2012 en: www. gentiuno.com/articulo.asp? articulo $=3536$

Vallersnes, O., Lund, C., Duns, A., Netland, H. \& Rasmussen, Y. (2009). Epidemic Of Poisoning Caused By Scopolamine Disguised as Rohypnol ${ }^{\mathrm{TM}}$ tablets. Clinical Toxicology, 47, 889-893.

Vanderhoff, B. \& Mosser, K. (1992). Jimson Weed Toxicity: Management of Anticholinergic Plant Ingestion. American Family Physician, 46, 526-530.

Voss, B., Thienel, R., Reske, M., Ute, H. \& Kircher, T. (2010). Cognitive Performance and Cholinergic Transmission: Influence of Muscarinic and Nicotinic Receptor Blockade. European Archives of Psychiatry and Clinical Neuroscience, 260, 106-110.

Wiebe, T., Sigurdson, E. \& Katz, L. (2008). Angel's Trumpet (Datura Stramonium) Poisoning and Delirium in Adolescents in Winnipeg, Manitoba: Summer 2006. Pediatrics Child Health, 13 (3), 193-196.

Yamamoto, S., Nishiyama, S., Kawamata, M., Ohba, H., Wakuda,T., Takei, N., Tsukada, H. \& Domino, E. (2011). Muscarinic Receptor Occupancy and Cognitive Impairment: A PET Study with MPB and Scopolamine in Conscious Monkeys. Neuropsychopharmacology, 36 (7), 1455-1465. 